\title{
An absurd re-ordering of game design through the possibilities of the database
}

Jenna Gavin

\begin{abstract}
This paper presents an alternative perspective in which to view creative generation and the practice of game design. A design research methodology incorporates sketch-based creative practice and the discussion of two theoretical frameworks: database and absurdity. The absurd is a rebellious embrace of a chaotic world, while the database is a new media structure for collecting, thinking and producing. These frameworks are competing yet complementary concepts that allow for exploration and ordering.

The creative practice described within embodies a database compulsion: the process of sketching many rough ideas instead of perfecting a single idea; the creation of a toolbox of general components, expressions and universal units; the creation of work that is non-linear, repetitive and/or random. Emerging from this structure, and yet rebelling from established order, is the notion of the absurd. Using Steve Hodges' notion of the digital absurd, a playful, absurd sensibility is proposed with the following qualities: unconvention; frustration; purposelessness; repetition.

The practice of sketching, coupled with the frameworks of absurdity and database, demonstrates a new media and interdisciplinary approach of playful ordering and reordering, re-classifying and reframing of game design and idea creation.
\end{abstract}

Keywords: Game design, absurdity, database, design research, creativity 


\section{Introduction}

The database is a technological manifestation of the human compulsion to collect; to possess; to know. It is a form of cultural and human collecting, as well as the ordering (and reordering) of items of significance. Leveraging the qualities of the database, one may proceed to generate ideas using a new media mindset. This mindset, which migrates away from conventional narrative and linear storytelling, presents an absurd attitude of repetition, frustration, purposelessness and 'unconvention'. Embracing an absurd attitude enables both designer and participant to challenge boundaries and assumptions, accept multiple meanings, and enjoy a playful purposelessness.

\section{Methodology}

Design is a complex process and "its open-endedness and reliance on creativity have made it a challenge for researchers to characterize and explain" (Edelson, 2002 , p. 108). Looking specifically at design as a process, the designer is someone who devises a set of actions in an attempt to "create desired outcomes" (p. 95).

This research explores ways of designing and generating ideas for games. By taking an experiential rather than goal-driven approach to game design, I hope to highlight areas that move beyond the boundaries of the status quo. Downton (2003) asserts that design discovery takes place mostly within the practice of designing; for this research, I have chosen an approach in which the creative practice of sketching parallels theoretical assertions.

Sketching is traditionally defined as a freehand drawing method (Kavakli, Scrivener et al., 1998). I interpret sketching as a multimodal practice of visual and interactive expression, while still maintaining vagueness, incompleteness, ambiguity and fluency. Not limited by medium, a sketch is therefore any activity that captures the essence of an idea and rejects the perfection of a finished product.

There are benefits to the sketch method. Since sketching captures only the essential qualities of an idea or experience, it enables effective communication for identifying characteristics (Kang, He, et al., 2005). Sketching provides an opportunity to restructure elements for the sake of creative discovery (Verstijnen, van Leeuwen et al., 1998), and is therefore able to produce "previously unseen and unexpected connections" (Langley and Jones, 1988, p. 177). Thirdly, sketching, without refining and without predefined intention, allows freedom from user-informed and bestpractice design methods.

The making process asserted an embrace of a ludic attitude. I playfully created, and created playful artefacts. Although I can propose a distinction between idea generation and game design, ideas and sketches inform other ideas and sketches, and I was not prepared to dismiss any creative expression in favour of a strictly defined notion of "game". Each sketch has the capacity to generate emergent meaning and worthwhile reflection for both designer and participant. Edelson (2002) describes "a parallel and retrospective process of reflection upon the design and its outcomes", which gradually produces a coherent theory (p. 106). True to Edelson's 
assertions, the frameworks of absurdity and database arose after I had considered the produced artefacts as a whole. This 'new knowledge' consequently informed the production of more artefacts.

While the sketches documented in this paper aid in discovering and illuminating the frameworks mentioned above, they cannot be artefacts that fulfil all theoretical speculations (this is best left to future research). As per Downton's (2003) assertions, for a theoretical paper about design, "there is no realistic expectation [to] exemplify a theory in giving an account of it" (p. 81). Further, the concepts I present are focussed on the craft of making, not scientific explanation; I therefore do not evaluate the ideas or artefacts empirically.

\section{Absurdity as Framework}

Typically, the notion of the absurd is understood as a logical impossibility (Hannay, 1991). Proposed by Albert Camus (1955), the philosophy of the absurd presents a rather larger human impossibility: the universe is chaotic and nonhuman, yet we seek truth, order and meaning from it.

Inherent to the philosophical absurd is an ethical stance. Absurdism is an appeal to the human experience, "as a methodological deconstruction of commonplace assumptions, including those regarding morality and politics" (Foley, 2008, p. 13). In recognising one's own absurd position, one realises the collective experience (Camus, 1992). With this solidarity comes a resistance to and rebellion of the status quo, of assumptions, of hierarchies, rules and conventions.

Hodges (2010) articulates that absurd principles-both the logical and philosophical-provide "an unusual and helpful perspective from which to view the emerging field of digital media" (p. x). An absurdist attitude allows us to take nonsense seriously and to accept multiple meanings (and indeed, no meaning) arising from artefacts and events. It provides a creative and humorous outlet to express the confusing, fantastical, contradictory, and the frustrating.

In The Digital Absurd (2010), Steve Hodges bridges the philosophy of the absurd with the aesthetics of the linguistic (literary and dramatic) absurd, as well as the logical absurd, to conceive of a contemporary absurdity: the digital absurd.

\section{The Linguistic Absurd}

In the 1960s, Martin Esslin coined the term "Theatre of the Absurd" to describe stage plays that, at first glance, appeared absurd; that is, they signalled no purpose, goal or objective (Esslin, 1960). Regarding Beckett, Adamov and Ionesco's work, Esslin observed a "deep sense of human isolation and of the irremediable character of the human condition" (p. 4). In a conventional play, "the action always proceeds towards a definable end" (p. 14). The audience is curious about what will happen next. Thus, the framework for understanding conventional works relies on a rational, popularly accepted view of life. In the Theatre of the Absurd, however, the purpose of the work is not clear or singular. In this instance, the audience is instead curious about what the play may mean. The audience is "compelled to puzzle out the meaning of what they have seen" (Esslin, 1960, p.14). 
The absurd play is a complex, multi-dimensional and contradictory work. It invites interpretation from multiple perspectives and demands intellectual attention. Absurdist literature and drama questions and undermines meaning, demonstrating meaninglessness of language that challenges the common ground of a shared reality. A barrage of nonsensical language mirrors the barrage of the inexplicable universe that humans experience. Our self-induced information age also overpowers us with volumes of communication and information. Nonsense and repetition hold strong positions in the absurd; they exasperate us, entertain us and create a sense of vastness and emptiness.

\section{The Logical Absurd}

There are glimpses of a more logical absurd within Beckett's work in the form of patterning and structure, specificity and optimism. The logical absurd contains repetitious elements, but avoids the overt approach of the linguistic absurd that invokes weariness and pessimism. Logical structures and "geometric arrangements" (Hodges, 2010, p. 109) hint at an underlying pattern or structure that may be present within the work, inspiring in us the hope of finding meaning.

\section{The Digital Absurd}

Using Camus' original absurdist philosophy, Hodges (2010) appropriates "this very broad notion to the more specific experience of the digital, translating the gap between human and world into a more specific form [i.e.] the gap between meaning and code" (p. 204). Hodges' focus is on the relationship between code and written language; however, I do not rule out the role of visual language in my interpretation of the digital absurd.

When experiencing absurd works of digital media, we are aware of the linguistic and visual surface phenomena, as well as of the underlying logical code that structures and dictates the digital experience. Our attention is initially occupied by the surface presentation (and the meaning we garner from it); however, following multiple interactions, absurd works tend to generate repetitions and possibilities that both "create and drain meaning away" (Hodges, 2010, p. 202). As we tire of the repetition, we begin to interrogate the structure or composition of the work in a bid to find patterns and function(s). However, the discovery of rules and logic, while providing us interim pleasure and understanding, will ultimately fail to explain or unify the meaning of the whole.

\section{A Database Compulsion}

In a general context, the term database is defined as "a collection of entries containing item information that can vary in its storage media and in the characteristics of its entries and items" (Oxford English Dictionary). Embracing both current and past definitions of the term datum, I propose a fluid use of the term: an item that varies, gives and informs. Umberto Eco (2009) describes the compulsion to collect and to list; to make sense of various aspects of our world. We keep these things in states of 'known' or 'possessed', grouping them to create our own knowledge or impose property boundaries upon the world: 
The list is the origin of culture. It's part of the history of art and literature. What does culture want? To make infinity comprehensible. It also wants to create order - not always, but often. And how, as a human being, does one face infinity? How does one attempt to grasp the incomprehensible? Through lists, through catalogs, through collections in museums and through encyclopedias and dictionaries (Umberto Eco, 2009).

With an influx of new media, the database form has become a dominant part of current culture that permits "the projection of the ontology of a computer onto culture itself" (Manovich, 2007, p. 42). Data structures (units) and algorithms (rules) make up this ontology. The computer and its users, in their binary way of understanding and ordering, reduce the world to discrete objects and qualities that can be modelled by data structures, in turn created and accessible by algorithms. Thus, impressions of the world are abstracted, simulated, duplicated, distorted, recorded, stored and recalled.

\section{Designing Through the Possibilities of Database}

I suggest, as a reflection on my own production, that there are a number of design methods that embrace a database compulsion:

- The process of sketching many rough ideas (instead of perfecting a single idea).

- The creation of a toolbox of general components, expressions and universal units.

- The creation of work that is non-linear, repetitive and/or random.

Such methods do not lend themselves well to fully realised game concepts; rather, their strength lies in the design possibilities they generate and the imaginative threads they offer to the designer or the participant.

I began my sketch endeavours by exploring tabletop board game mechanics (Figure 1 and Figure 2). I played with paper, card and wood components, without the purpose of creating a game, instead creating abstract actions and movements that seemed "board game-like". The result is a collection of abstract actions that are available to employ in specific game ideas. Rough sketching generated a collection of universal units.

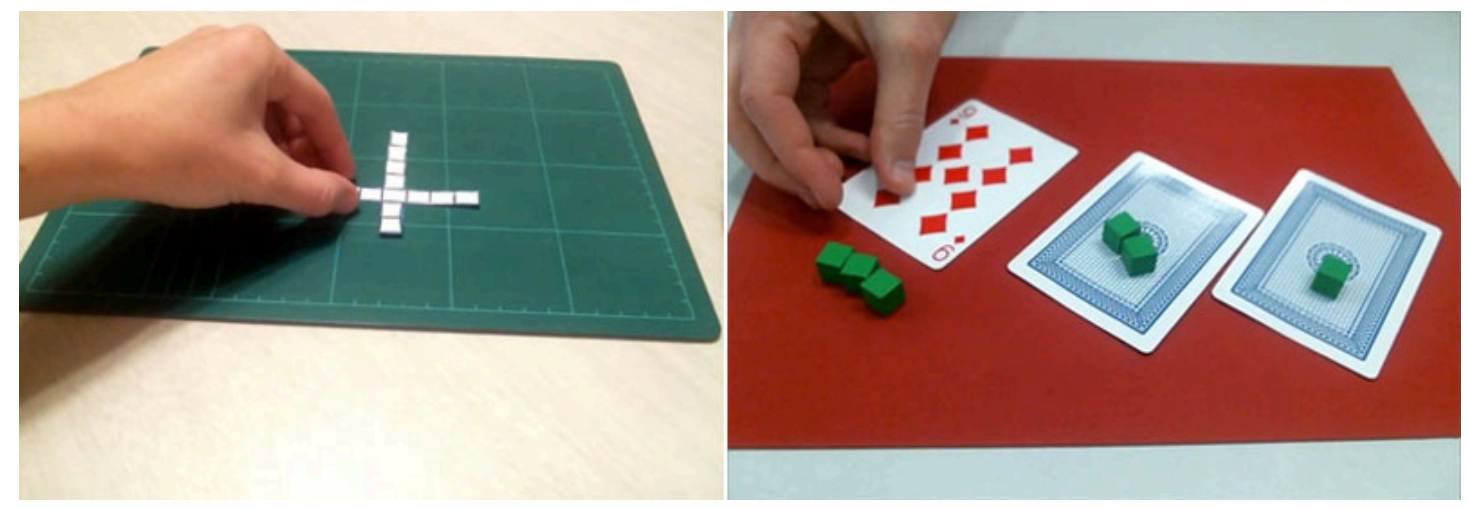

Figure 1. Tabletop game actions: general. 

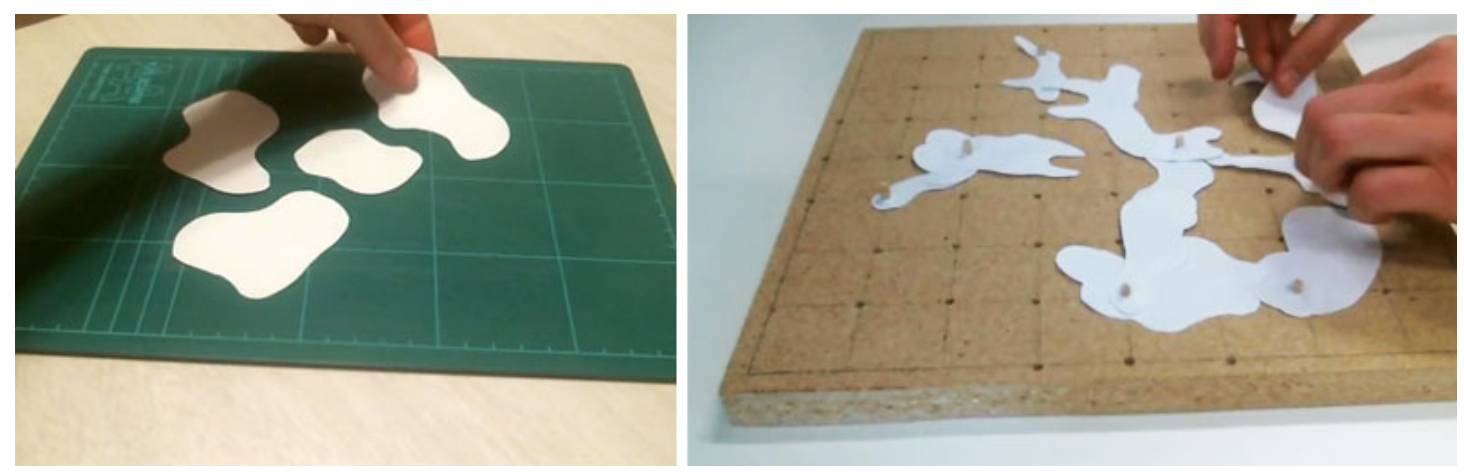

Figure 2. Tabletop game actions: puddles.

The purpose of the above design experiments was to establish a utility-box of mechanics that could be applied to future game inspirations. This was a straightforward approach to enhancing my game design brainstorming toolbox, although I was unaware of my own database tendencies at the time of designing. In the brainstorming experiment described in the next paragraph, I was largely aware of the collection I was creating, although the purpose was less clear.

Initially, and without any more purpose than to automatically, intuitively sketch, I sat and drew. I allowed my hand to move freely and repetitively while drawing abstract lines on paper. Continuing the habit over the course of a month, I amassed a collection of pencil and pen drawings (Figure 3). I began to see the drawn sketches as idea generators. They were spaces, places, emotions, moods, architectures.

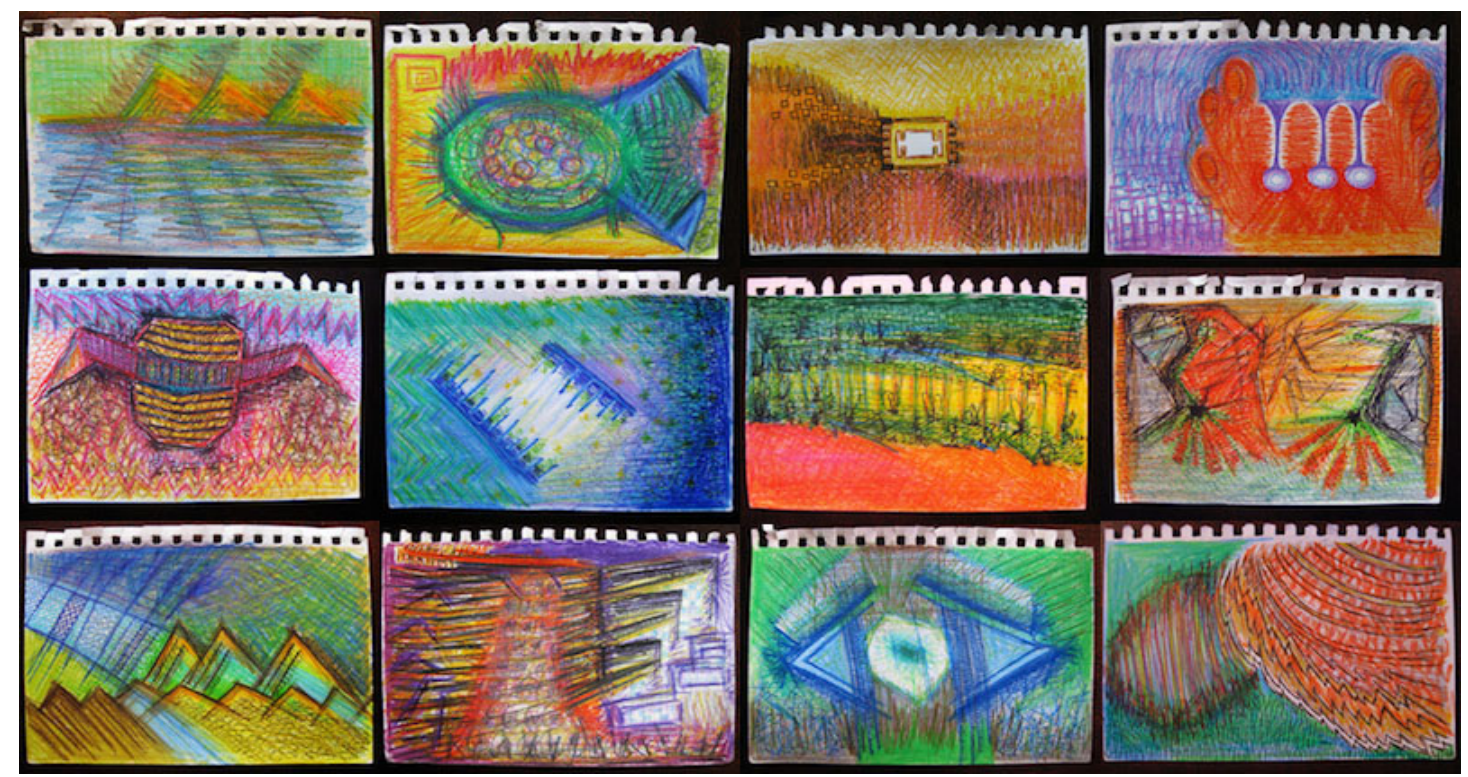

Figure 3. Drawings on notebook paper.

Mesh (Figure 4) was an attempt to create traversable digital environments. Following an OpenFrameworks tutorial by Michael Hadley, I was able to create vector meshes based on the colour data of a sketch from the drawn collection above. I was able to move around inside a virtual 3D environment that had been created by the data from a completely different 2D landscape. This process, of manipulating and re-ordering sketches to create new sketches, is capable of generating new possibilities and moving between mediums. 


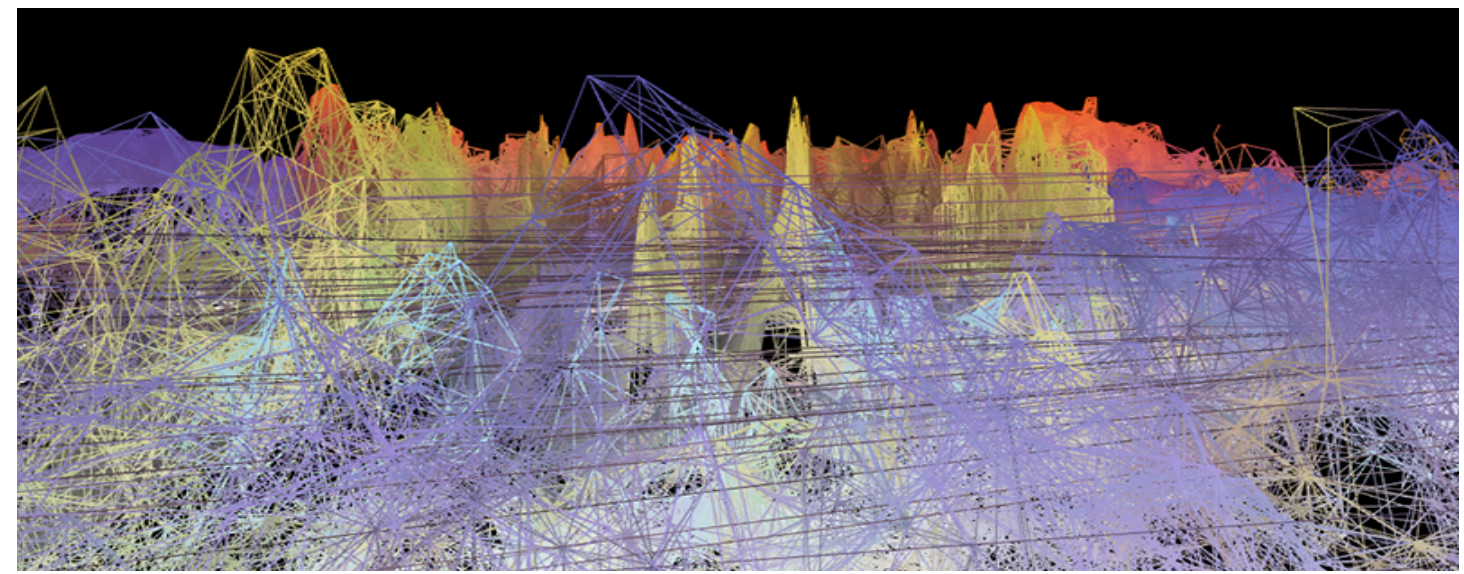

Figure 4. Mesh.

\section{The Narrative and Non-linearity Found in Database}

The database presents an order that can be read, like a narrative, in multiple ways. Traditionally, narrative is a linear, cause-and-effect sequence of events that is not ordered by anything but a timeline. Database supports narrative, but is not narrative in and of itself. Manovich (2007) reasons that database and narrative forms emerge as humans strive to make meaning from the world. They are "two competing imaginations, two basic creative impulses, two essential responses to the world that have existed long before modern media" (p. 51).

New media favour database forms. These may be complex data structures, but Manovich suggests that from a user's point of view, databases simply "appear as collections of items on which the user can perform various operations: view, navigate, search" (p. 81). If we redefine our understanding of narrative to accommodate new media, the experience becomes "the sum of multiple trajectories through a database" (p. 46). In new media, the database is the centre of the creative process and multiple interfaces can be constructed to interact with it. This opens up possibility, what Manovich terms "variability".

Roots (Figure 5) is a rudimentary Processing sketch modelled on plant roots. The roots split and grow to create a complex mess of lines. Each line has a random downward trajectory, which creates a unique sketch on every occasion. Admittedly, the "interface" and content is too simple to allow for proper narratives to emerge.
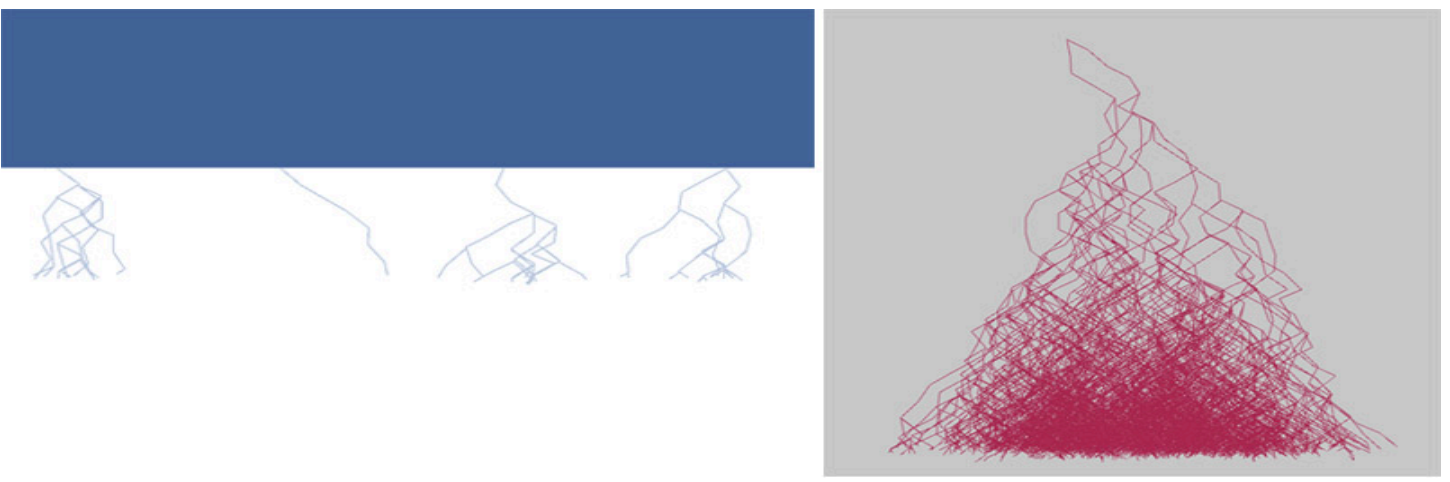

Figure 5. Roots: early explorations. 
Closer to Manovich's (2007) descriptions are the next set of sketches, which expose the database structure and permit (absurd) narratives to emerge.

\section{Exposing the Database Structure}

Expanding on the initial Roots sketch, I built a basic navigation system on the root lines, which the participant could use to produce words that create a spatially related and randomly generated poem (Figure 6). In a non-linear fashion, words are pulled from a linear piece of work in a file behind the scenes.
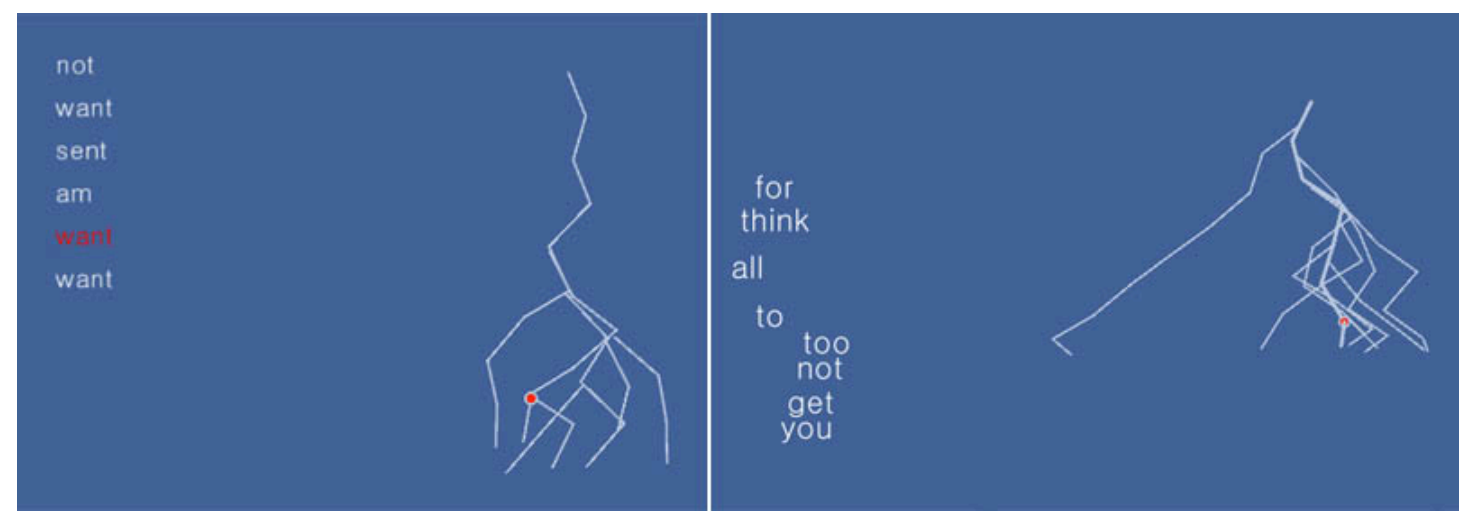

Figure 6. Roots: poem explorations.

Food Pairings (Figure 7) is an OpenFrameworks application that presents pixel manipulation with combinations of foods and flavours. It plays with possibility to generate a sensory scene within the user's mind. With repeated use, its structure is revealed as an obvious interface over database elements. Each time the space bar key is pressed, a new story is generated by changing the vegetables, odour, and country. The stories have the potential to evoke a meaningful response from the user, as she imagines the particular sentences in her mind.
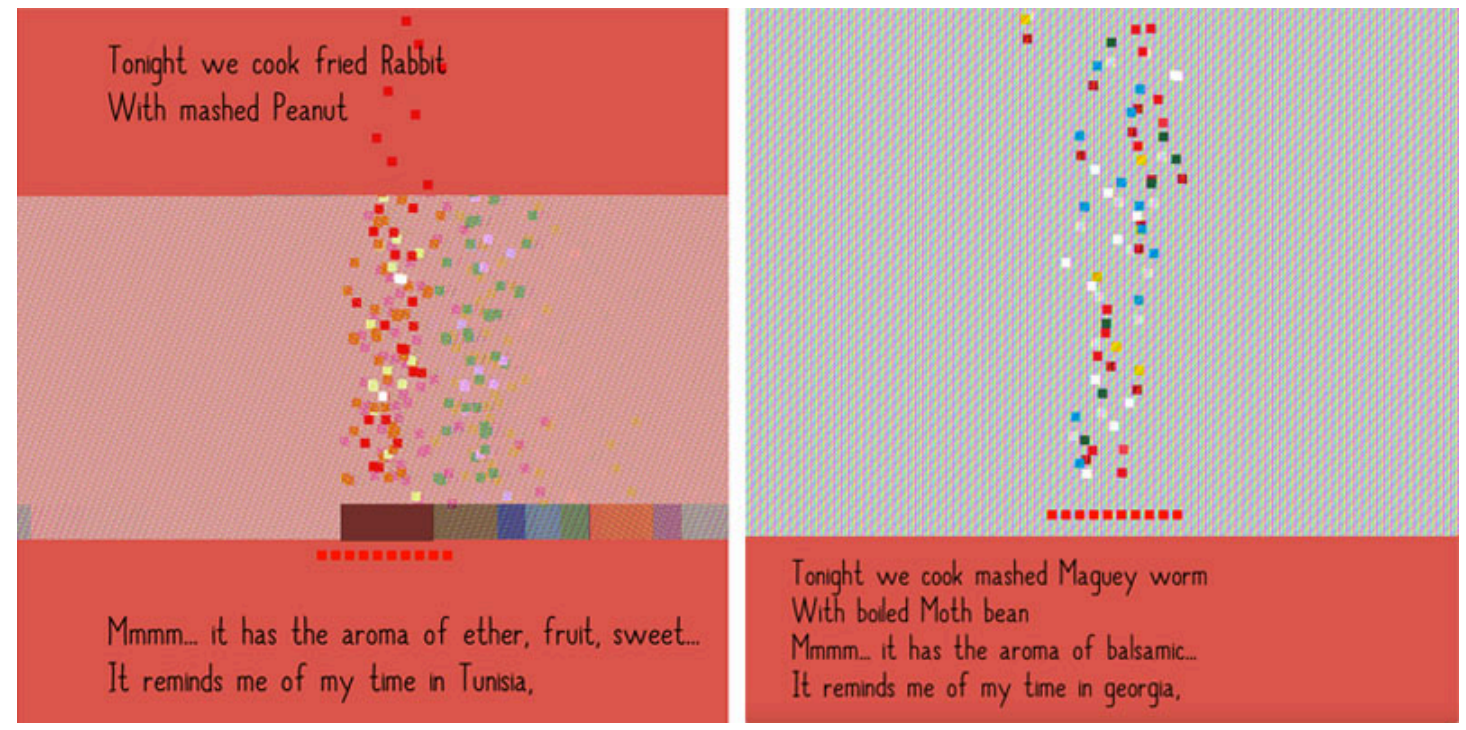

Figure 7. Food pairings. 
Wisdom (Figure 8) is a Processing application that generates a simple sentence of "advice". It is structured in the mode of online generators that produce, for example, random names, insults and "tweets". Wisdom draws on three lists: imperative; action; object. The idea arose from a conversation with a friend over dinner. The intention is that the user may garner a personal meaning from the randomly generated phrase, hopefully with a reflective perspective.

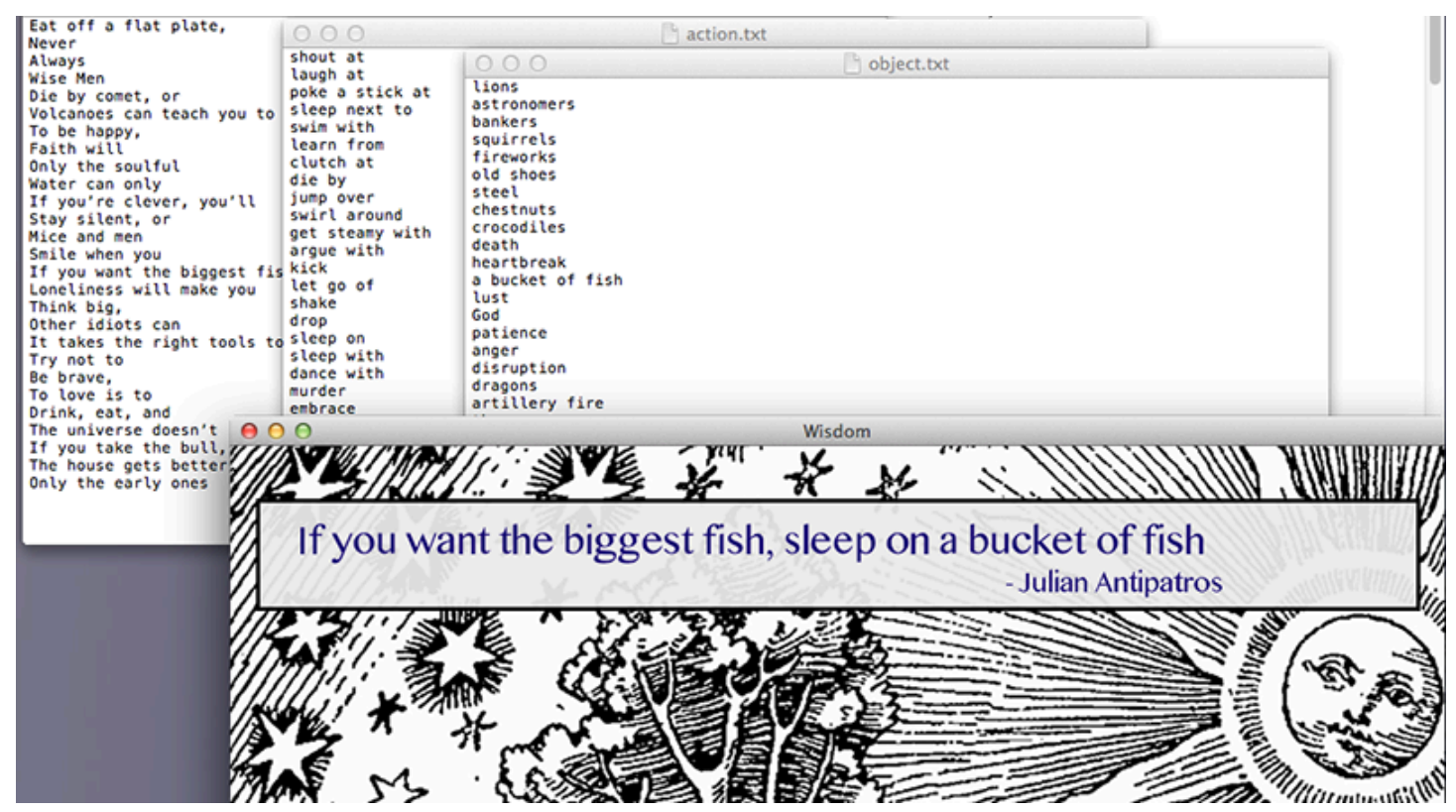

Figure 8. Wisdom.

\section{The Space of Possibility}

A game design implies future action in the form of possibility. Salen and Zimmerman (2004) define the space of possibility as "the space of all possible meanings which can emerge from a game design" (Chapter 6, p. 11):

The space of possibility is designed (it is a constructed space, a context), it generates meaning (it is the space of all possible meanings), it is a system (it is a space implied by the way elements of the system can relate to each other), and it is interactive (it is through the interactive functioning of the system that the space is navigated and explored) (Chapter 6, p. 11, Salen \& Zimmerman, 2004).

Game designers can never fully predict or control the experience and actions of players, and not only because games exist in a real-world context. Fullerton et al. (2006) explain that game design has unpredictable results, due to what Salen and Zimmerman (2004) refer to as a "second-order design problem". In other words, complex systems create emergent, unforeseen behaviours. To counter this unpredictability, Salen and Zimmerman recommend an iterative design process that includes prototyping and playtesting. If one is to publicly present a game and expect it to be balanced, iterative design is essential.

Dormans (2011) acknowledges that designing for emergence is a complex process. He suggests using simpler rules, ones not designed to simulate a complex reality, but 
rather to capture the essence of a system. Rusch and Weise (2008) advocate for complex ideas to be designed into games in abstract ways.

\section{Abstraction, Realism and Perfection}

To abstract is to express the virtuality of nature, to make known some instance of its manifold possibilities, to actualise a relation out of infinite relationality (Wark, 2004, p. 2).

Like the abstraction of a database and the abstraction inherent in sketching, abstraction in game design distils an idea into identifiable characteristics. When a designer sets out to create a game about something, the game mechanics become a representation of the actions being simulated or modelled. Game designers are compelled to consider abstraction due to the nature of their medium.

Looking at game design through an artistic lens provides valuable insights into the concept of abstraction. Art scholars understand that abstraction is inherent in a work of art because it is a representation (Zimmer, 2003). A piece of art is a subjective expression of reality, a human extraction, a bridge. Fundamentally "a work of art functions as a merging agent between a known world and a perceived world" (Gortais, 2003, p. 1248). In terms of abstraction in art, Zimmer asks: "how can you decide what features should be exaggerated? The answer is: find the things that sum up the essence of your subject. And these are, as it is in all caricature, the things that are furthest from some sense of normality, some anchor point" (p. 1288).

The game designer can step away from realistic (iconic) representation and focus on the essentials of the experience. By abstracting, using indexical and symbolic representation, "games will have fewer details and thus usually fewer inconsistencies to distract the players. Players will have more room for personal interpretation making the experience more relevant" (Dormans, 2011, p. 627). Not only do players have more room for personal interpretation this way - the game designer as artist also has more room for personal expression.

Cuckoo (Figure 8) is a sketch that is representative in nature. It consists of a collection of cards intended as the basis for a tabletop game. The inspiration for this set of cards came from wanting to design a game about the cuckoo bird as a brood parasite. The main idea that I wanted to represent was the stealthy attempt to disguise a cuckoo egg in a nest full of other eggs. 


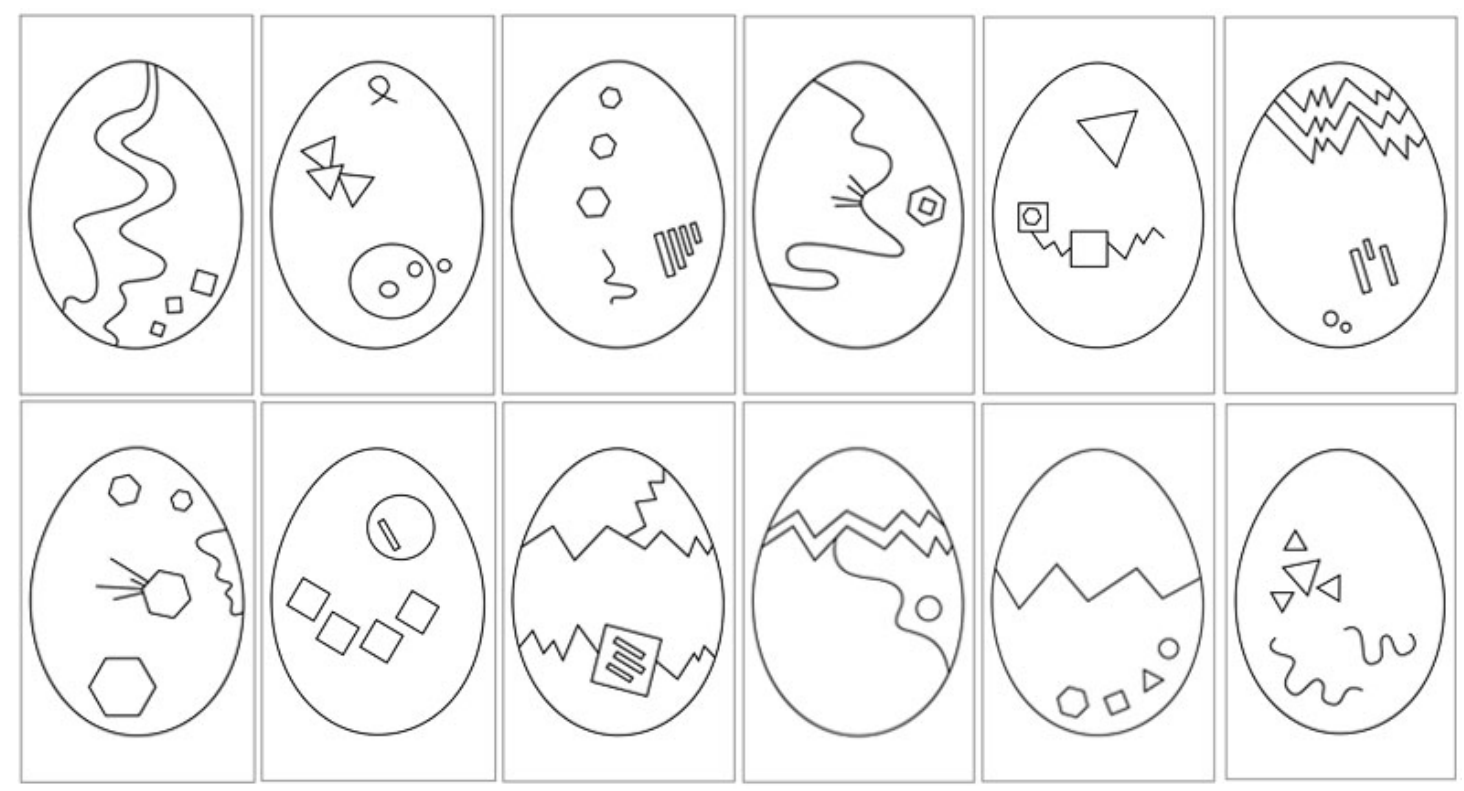

Figure 9. Cuckoo cards.

In trying to capture reality through a representation, "imitation is doomed to failure. Imitation is always disappointing because it is less than the real object" (Gortais, 2003, p. 1241). Striving for perfection and completeness does not sit well within an absurd and creative framework, although it is precisely the ideal of completeness that the database compulsion tries to achieve. By recognising that perfection is an unreachable ideal, the designer can consciously turn to rebellion and disruption, while still engaging with possibility.

\section{Arcade of the Absurd}

Building on the concept of the "Theatre of the Absurd", Sam Posner (2011) imagines for games an "Arcade of the Absurd". His article on the topic explains the concept of absurdist art and absurdist video games:

The lack of meaning to human existence does not mean that life is not worth living. Accordingly, absurdist artists believe that the struggle to perfect art to some ideal is absurd - as there is no intrinsic value, there is no "perfect art." Therefore, the absurdist artist strives not for unreachable perfection but to mirror the absurd human condition... Consequently, absurdist games upset the evolution of typical games, twisting mechanics that [have been] accepted as part of a genre's progression toward perfection [and by doing so] challenge players' conception of a "well made game" [while furthering] a virtual absurdist philosophy: one should game for the sake of gaming (Posner, 2011).

Like the absurd stage play, the absurd game is unclear and contradictory. The player is made to wonder what the game may mean. Thus, the game is open to interpretation; the rejection of genre and convention becomes an opportunity for cross-pollination, for both designer and player to explore boundaries and broaden experiences. 


\section{Struggle, Challenge, Revolt}

An iterative design process will refine the intended outcome of a game's design. However, without iteration, a game designer may still capture an essential idea in a functional (albeit potentially flawed) form. In sketch-based practice, capturing trumps refining. Indeed, a sketched game design should struggle with a new idea, as "sketching is about creating or capturing possibilities" (Newall, 2012, p. 32). When struggling with a new idea or a new thing, the initial experience may be difficult, boring, nonsensical or frustrating.

Furthermore, I suggest that difficult sentiments are an acceptable outcome when exploring ideas within the game medium. New ideas may necessarily disrupt or subvert the game medium's conventions of efficiency, usability and what might be considered "fun". Addressing difficult themes in her non-digital game series, Brenda Brathwaite (2010) had to step "beyond the commercial possibility space" (p. 313) in terms of game design. Using narrative and mechanics not to entertain, but to explore the human experience, she has instilled in her games a sense of artistic freedom comparable to that of other mediums.

\section{Dialogic Game Design}

Douglas Wilson (2012) suggests alternative possibilities for game design. Wilson suggests "dialogic design" as "an attempt to kindle challenges or dares around design objects" (p. 55). He uses the art forms of crime novelist Agatha Christie and performance artist Marina Abramović to highlight his point:

If dialogic design can be understood as a battle of wits and willpower between creator and audience, then Christie and Abramovic explore the two extremities of that confrontation - Christie's work as a confrontation of wits, Abramović's work as a confrontation of willpower (Wilson, 2012, p. 42).

This attitude encourages the game designer to subvert, disrupt, frustrate, tease, and confuse. It treats the audience as intelligent and curious; it invites the player into a universe, but does not spoon-feed them the rules and conventions of the universe. The reward for the participant comes in persevering, uncovering meaning within both themselves and the work. The reward for the designer is the pleasure of crafting a meaningful and challenging experience.

Games, like absurd literature, are capable of a mocking and a light-hearted attitude. By rejecting convention and genre, by refusing to play by the medium's established rules, a game can become self-aware and open up a dialogue between designer and player.

I have engaged in dialogic game design using the sketch Jaguar Returns (Figure 10), a puzzle game built in OpenFrameworks. The work emerged from a digital, interactive expression of the 24 Reasons poems, and continued as I found inspiration in The God's Script (1949) by Jorge Louis Borges. In this story, a prisoner tries to decipher the language of God-which he has found on the coat of a jaguarbelieving it will set him free. He is consumed by visions and dreams within dreams. $\mathrm{He}$ is lost, yet obsessively hopeful that he will unravel the enigma that he has 
discovered (or created for himself). From "the tireless labyrinth of dreams" (p. 206), the prisoner awakes to know the universe and the answer to freedom: a formula of 14 random words that, if spoken, will make the prisoner all-powerful.

Jaguar Returns is thematically influenced by Borges, with a liminal mood, a symbolic, fantastical space, and a confrontational attitude. Abstract rectangular structures present possibility spaces that can be moved through, forming a narrative-like experience. The player can move between 14 different "dreams", each a separate sketch, through which I attempted a distinct game mechanic or puzzle. On completion, each puzzle reveals one of 14 random words. Together, all the words unlock the "end of the game". The dreams themselves are comprised of simple geometric shapes, lines, and movements. On a technical level, I was simply trying to create a variety of mechanics and game goals using quick and rough code.

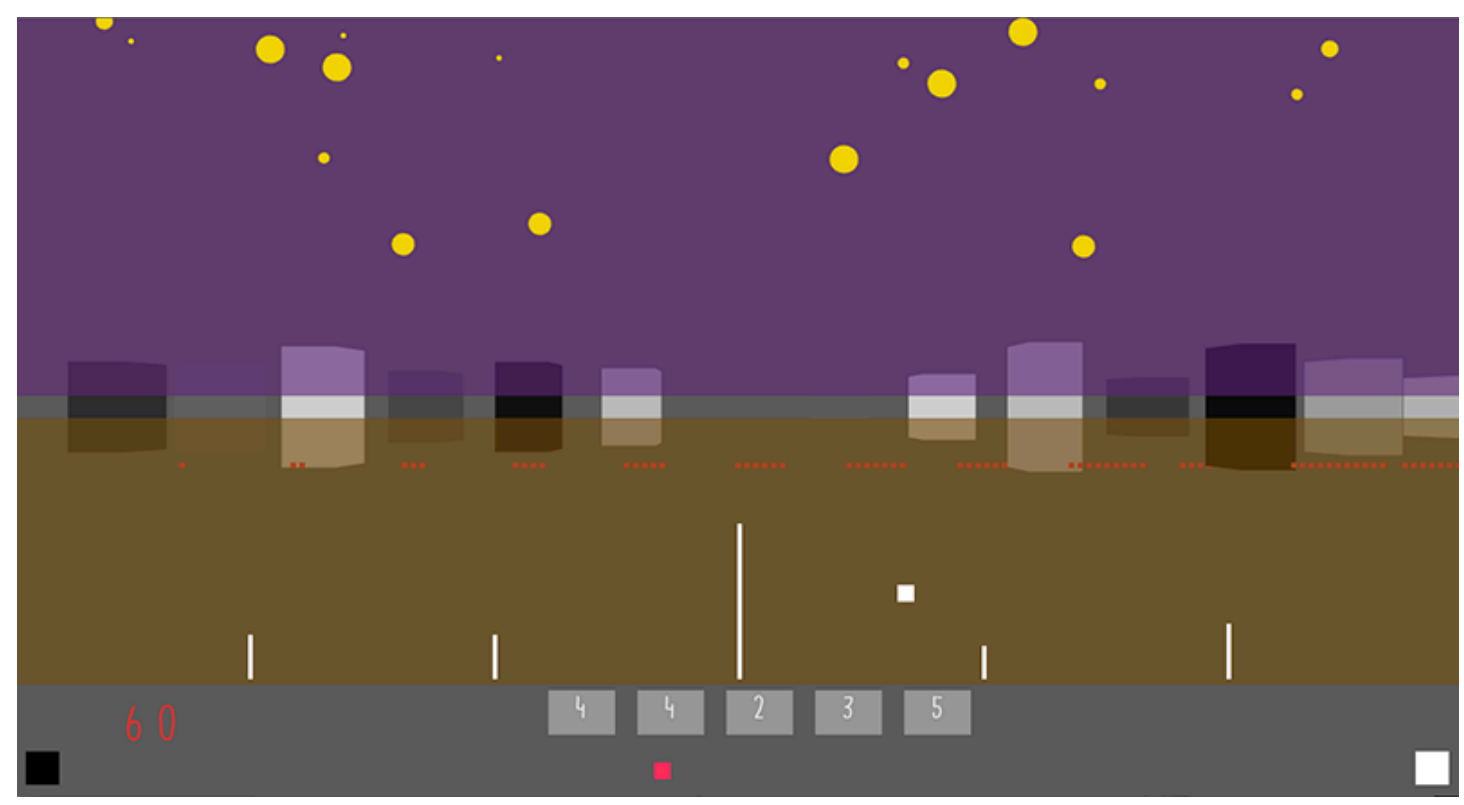

Figure 10. Jaguar Returns.

I found that by using simple shapes, not only was the process of sketching quicker, the abstraction allowed for personal interpretation and personal expression. This virtual world has no instructions or explicit narrative; it does not explain itself. Players must seek out their own meaning and formulate their own narrative. At the outset, these activities present a challenge for the player. Continuing with a confrontational attitude, I began to actively attempt to create puzzles that would frustrate, tease, and intrigue the intended player. 


\section{A Playful and Interactive Absurd Sensibility}

With the discussion on database and the absurd, together with the game-oriented discussion in this chapter, I can begin to illustrate a picture of a playful and interactive absurd sensibility. I suggest that the playful and interactive absurd work:

- Does not strive for perfection or to provide an absolute explanation

- Does not express a conventional interpretation of reality

- May invoke frustration, intellectual thought, weariness and/or bewilderment

- May employ repetition, contradiction, the unconventional and/or the illogical

- Holds an attitude of rebellion and disruption

- Has a sense of humour and/or self-awareness

- Lacks unification in terms of its linguistic or visual meanings and its underlying structure

Condensing the above into four broader absurd qualities that are present in the game medium, I propose the following merits as they pertain to game design: unconvention, frustration, purposelessness, and repetition-outlined in Table 1.

Table 1

Four absurd qualities present in the game medium

\begin{tabular}{|l|l|}
\hline Unconvention & $\begin{array}{l}\text { In what can loosely be termed a 'revolt' against the status quo of } \\
\text { commercial game design, unconvention is an attempt at infiltrating } \\
\text { new territory and pushing boundaries. This includes pushing against } \\
\text { structural and thematic conventions. }\end{array}$ \\
\hline Frustration & $\begin{array}{l}\text { Frustration stands in opposition to "frictionless design". The } \\
\text { consensus within the interaction design field is to render interactions } \\
\text { and experiences efficient, smooth, and transparent. However, to play } \\
\text { is to intentionally embrace inefficiencies and artificial conflict (Salen } \\
\text { and Zimmerman 2004, Chapter 7, p. 11); the struggle is not meant to } \\
\text { be overcome, but to be embraced. The designer can enjoy frustrating } \\
\text { a player's expectations of genre and interface. Challenges, puzzles } \\
\text { and interactions should frustrate a player! }\end{array}$ \\
\hline Purposelessness & $\begin{array}{l}\text { A wider sense of frustration arises from a player's attempt to } \\
\text { determine meaning and genre in a work that exudes, at least on the } \\
\text { surface, purposelessness. Games are typically defined by an } \\
\text { overarching goal, that is, a "quantifiable outcome". They typically } \\
\text { follow popularly accepted conventions that aid in situating meaning. }\end{array}$ \\
\hline Repetition & $\begin{array}{l}\text { A staple in the literary absurd, repetition creates a sense of vastness } \\
\text { and emptiness. It implies possibility and an underlying order, but also } \\
\text { creates weariness and destroys suspension of disbelief. Sometimes, } \\
\text { however, the absurd holds its pose for a fraction too long and the } \\
\text { work reveals an outer discourse to the participant through its } \\
\text { ridiculous repetition. }\end{array}$ \\
\hline
\end{tabular}




\section{Exploration and Order}

Considering the frameworks of absurdity and database, it may be helpful to draw from Kant's freeplay of the faculties. As Kovach (1993) explains this notion: "two faculties of mind; that which explores the possible (the imagination) and that which orders the given (the understanding)" (p. 166). Both the database and the absurd allow for exploration and order.

We can imagine that the database is the ordering of chaos created by the universe. Storing and structuring items in a database presents a way for creating meaning out of a seemingly meaningless world. Manovich's (2007) new media mindset implies we reduce the world to discrete objects and qualities; we categorise and simplify to make sense of a complex reality. The database is also a structure for exploring possibility. The units in a collection (data) are items that vary, give and inform. Separately and as a whole, they allow for the construction of meaning.

An absurd sensibility emerges from the repetitive, non-linear and multiplex nature of the database. Paradoxically, the absurd also disrupts order created by society. Through the unconventional and the challenging, the absurd allows for an exploration of multiple interpretations and experiences outside of the status quo. The absurd orders itself with logic and repetition, offering the hope of finding meaning in underlying structure, but never delivering complete resolution.

\section{Concluding Thoughts}

Game designers must employ both imaginative and rational faculties to craft playful artefacts. From one end of the spectrum to the other, a game designer explores and orders. The database offers a structure in which to generate possibilities, collect ideas, and record meaningful cultural and personal objects. Absurdity, meanwhile, encourages a playful embrace of the ordinary, the nonsensical, the purposeless, and the fantastical.

Absurdity introduces a rebellious and boundary-pushing attitude into game design, which brings with it the chance to touch on very human experiences. With an absurdist point of view, one may challenge commercial and social hierarchies. (In a critical discourse such as this, it is not without some irony that I recognise that this absurdist framework sits inside academically rigid conventions)

This research may prove useful to anyone looking for alternative and more absurd approaches to creative making, an interdisciplinary approach toward playful generation. Perhaps crafting purposeless, frustrating, and unbalanced "game-like" artefacts is not a model for popular success. Yet, these types of endeavours deliver meaningful experiences, generate original thoughts and ideas, and provide a foundation for new syntheses. 


\section{References}

Camus, A. (1955). The myth of Sisyphus, and other essays. Random House LLC.

Dormans, J. (2011). Beyond iconic simulation. Simulation \& Gaming, 42(5), 610-631, doi:1046878111426963.

Downton, P. (2003). Design research. RMIT Publishing.

Eco, U. (2009). SPIEGEL Interview with Umberto Eco: We Like Lists Because We Don't Want to Die/Interviewer: L. Gorris \& S. Beyer. SPIEGEL Online.

Edelson, D. C. (2002). Design research: What we learn when we engage in design. The Journal of the Learning sciences, 11(1), 105-121.

Esslin, M. (1960). The Theatre of the Absurd. The Tulane Drama Review, 4(4), 3-15.

Foley, J. (2008). Albert Camus: from the absurd to revolt. Acumen.

Gortais, B. (2003). Abstraction and art. Philosophical Transactions of the Royal Society B: Biological Sciences, 358(1435), 1241-1249.

Hannay, A. (1991). Kierkegaard: arguments of the philosophers. Routledge.

Hodges, S. (2010). The digital absurd. Georgia Institute of Technology.

Kang, H. W., et al. (2005). Interactive sketch generation. The Visual Computer, 21(810), 821-830.

Kavakli, M., et al. (1998). Structure in idea sketching behaviour. Design studies 19(4), 485-517.

Kovach, V. (1993). Judgement as play: revealing analogies between aesthetics and ethics. ResearchSpace@ Auckland.

Langley, P. \& R. Jones (1988). The nature of creativity: Contemporary psychological perspectives. R. J. Sternberg, CUP Archive.

Manovich, L. (2007). Database as Symbolic Form. Database Aesthetics: Art in the Age of Information Overflow. V. Vesna. Minneapolis, MN, University of Minnesota Press: 39-60.

Newall, K. (2012). Sketching as a Methodology for Creative Practice. AUT University.

Oxford English Dictionary. (2015). Oxford English Dictionary. from http://www.oed.com/

Posner, S. (2011). Arcade of the Absurd. from http://nightmaremode.thegamerstrust.com/2011/06/24/arcade-of-the-absurd/

Salen, K. \& E. Zimmerman (2004). Rules of play: Game design fundamentals. MIT press. 
Verstijnen, I. M., et al. (1998). Sketching and creative discovery. Design studies 19(4), 519-546.

Wark, M. (2004). Hacker Manifesto version 2.0.

Wilson, D. E. (2012). Designing for the Pleasures of Disputation-or-How to make friends by trying to kick them!. IT University of Copenhagen, Innovative Communication.

Zimmer, R. (2003). Abstraction in art with implications for perception. Philosophical Transactions of the Royal Society of London B: Biological Sciences 358(1435), 1285-1291.

\section{List of Figures}

Figure 1. Tabletop game actions: general. 5

Figure 2. Tabletop game actions: puddles. 6

Figure 3. Drawings on notebook paper. 6

Figure 4. Mesh. 7

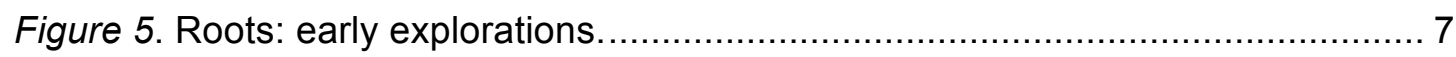

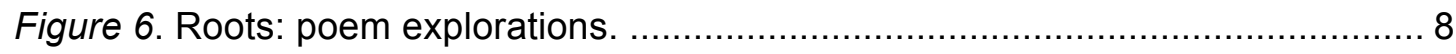

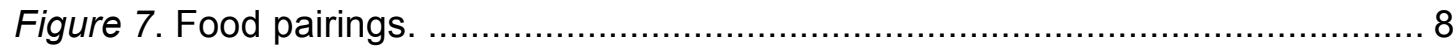

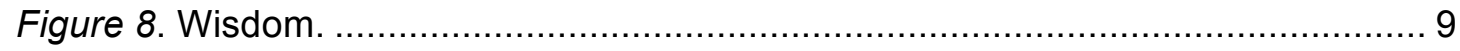

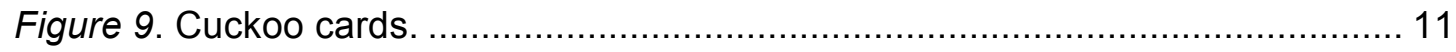

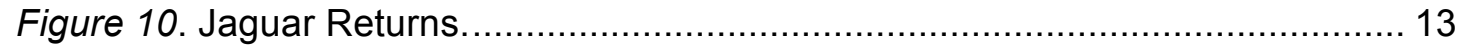

\section{List of Tables}

Table 1. Four absurd qualities present in the game medium 14 


\section{About the Authors}

\section{Jenna Gavin}

Jenna Gavin is a digital media artist and game designer who creates playful interactions with language, data and form. Her strength lies in crafting unique user experiences through code and objects. Her eight-player game "Word Wars" has been played at the Auckland Art Gallery, the Semi-Permanent Research Lab and the PopUp Indie Games Arcade. Her work has also featured on the Digital Art Live screen in the Aotea Centre and the Auckland War Memorial Museum. She is currently concluding a Masters of Creative Technologies at Auckland University of Technology, with practice-based research that explores a playful and absurd approach to game design.

Contact: jlgavin@gmail.com 


\section{The Journal of Creative Technologies (JCT)}

https://ctechjournal.aut.ac.nz

ISSN: $2230-2115$

Colab, Auckland University of Technology, New Zealand

Creative Commons Attribution 4.0 International License (CC-BY)

All articles published in The Journal of Creative Technologies (JCT) from Issue 4 onwards are licensed under a Creative Commons Attribution 4.0 International License (CC-BY). The copyright of the material remains with the author(s), and third-parties are granted permission to use, shared, and adapted the material, provided the original work is appropriately attributed.

The Journal of Creative Technologies (JCT) is an online, open access, peer-reviewed journal for the publication of research and innovation about new technologies, creative practices, and critical theories. The journal aims to explore applied, methodological and theoretical perspectives on emergent technological platforms and strategies through thematically focused issues.

$J C T$ is a research communication platform published by Colab at the Faculty of Design and Creative Technologies, Auckland University of Technology. 